\title{
Yoga and Health
}

\author{
Ivan Uher*, Iveta Cimbolaková, David Kaško and Zuzana Küchelová \\ Institute of Physical Education and Sport, Pavla Jozefa Safarik University, Slovakia
}

Submission: May 18, 2018; Published: October 16, 2018

*Corresponding author: Ivan Uher, Institute of Physical Education and Sport, Pavla Jozefa Safarik University, Košice, Slovakia, Email: ivan.uher57@gmail.com

\begin{abstract}
The background of yoga has its origin in ancient Indian philosophy. In contemporary society we can observe many schools or types of yoga. Each having its own specific emphasis regarding body postures, breathing techniques, relaxation technics, and meditation practices. That nurture awareness and state of consciousness. The application of yoga as a therapeutic means, take advantage of the number of psychological and physiological benefits. Yoga exercises may increase man flexibility, strength, coordination while the breading and meditation practices may silent the mind, hence evoke greater awareness and diminish anxiety, reduce distress, and thus enhance quality of life. Despite of growing body of clinical research on the therapeutic effects of yoga, there is still a lack of evidence regarding its clinical validity for number of symptoms and health conditions. In some research studies we can observe inconsistent evidence with some reporting positive effect of yoga interventions, but some are less conclusive. In our review we summarize the current evidence on the clinical effect of yoga intervention on various factors of physical and mental health.
\end{abstract}

Keywords: Fitness; Yoga; Health; Physiology; Psychology

\section{Yoga and Mental Health}

Number of research studies outline plausible effects of yoga interventions on inner health. However, not all studies used adequate or congruent instrument to evaluate the overall effect of yoga intervention on mental health. Even though not all studied apply same tools to evaluate stress, they actually prove that yoga may limit stress level as impressive as other actively regulated interventions like cognitive behavioral therapy, relaxation [1]. When we evaluate the effects of yoga on fatigue in various medical conditions such as sclerosis multiplex, cancer, chronic pancreatitis and asthma we can found overall small but plausible effect [2]. The same effects we can observed in healthy people [3]. Number of studies investigating effects of yoga on anxiety describing beneficial effects when compared with passive and active controls [4]. The study results investigating effects of yoga on depression have revealed mixt results [5]. Indeed, there is an exigence to conduct more research studies with sound methodological platform on larger sample size to determine whether there is substantial justification to consider yoga as a treatment of depression.

\section{Yoga and Physiological Systems}

Most studies result pointing out to the conclusion that yoga has positive effect on sympathetic and parasympathetic activation and cardiovagal function [6]. We have alleged claims that yoga can positively enhance modification in sympathetic activation of cardiac and vagal function, and also contributes to the autonomous neural system homeostasis primarily by sympathetic to parasympathetic activation [7]. Literature review on the effect of yoga on cardiovascular endurance reporting significant improvements in overall cardiovascular endurance of young subjects who were given varying periods of yoga training for extended period of time [8]. Studies investigating the effects of yoga on blood pressure and hypertension report a reduction of systolic and diastolic pressure. However, there are several noted potential biases in these studies (i.e., confounding by lifestyle or others) which makes it difficult to detect an effect specific to yoga [6]. Some authors found small, insignificant improvements of systolic and diastolic blood pressure in favor of yoga when compared to no treatment [1]. When we evaluate yoga's effects on lung function in healthy volunteers and patients with bronchitis and asthma we can conclude that in healthy volunteers practicing yoga, there are reported improvements of selected pulmonary function with inspiration and expiration breathing control techniques, particular body postures and relaxation methods [8]. In another study on patients with asthma we can observe improvements in peak expiratory flow rate, medication use and asthma attack frequency [8]. Systematic reviews examining the effects of yoga on risk indices associated with insulin resistance syndrome reported postintervention improvement in various indices in adults [6]. However, we have to take into consideration that the results varied by population (healthy adults, adults with cardiovascular disease risk, adults with type 2 diabetes, etc.) and study design. Studies evaluating yoga in comparison to medication intervention showing significant subjugation of fasting glucose with type 2 diabetes mellitus. We can conclude that differences in study populations, and interventions can stand out as a possible explanation for the observed heterogeneity of results. When we observe effects of yoga on musculoskeletal function, chronic 
pain conditions, and pain associated disability (lower back pain, arthritis) all of these studies reported positive effects with respect to pain $[9,10]$. Moreover, studies that included yoga for the treatment of headache- migraine, hemodialysis, irritable bowel syndrome, labor pain, etc., All of these studies reported positive effects in favor of the yoga interventions [9]. Despite some study limitations, there is evidence that yoga may be useful for several pain-associated disorders. Thus, well-design large scale studies are needed to verify these findings.

\section{Health Benefits of Yoga and Exercise}

A growing body of research evidence supports the belief that certain yoga techniques may improve physical fitness [11]. While exercise has been shown to definitely improve parameters of fitness, the fitness effects of yoga have only been examined in handful of studies $[12,13]$. Yoga seems to provide many of the benefits typically associated with exercise [11]. It is possible that differences in fitness outcomes found in the comparison studies of yoga and exercise might not have been found if exercise were compare to the more vigorous forms of yoga. The differences that have been found between yoga and exercise interventions may be a result of how the two differ in their effects upon the hypothalamic -pituitary-adrenal axis and the sympathetic nervous system [14]. To make distinct differences between yoga and conventional exercise with the current research has proven to be difficult. In one study significant increases of strength, muscle endurance, flexibility, and $\mathrm{VO}_{2}$ max occurred in 10 healthy volunteers after 8 weeks of biweekly asana and pranayama classes [13]. In one study [15], sun salutation practice had led to decrease in body weight and \% body fat and increase in \% lean body mass. Further, practicing yoga for $30 \mathrm{~min}$ a day increased MET to 2.5 in females. But inclusion of "Sun salutation" in the session increased the MET to 3.74 [16]. When we look on older subject with respect to physical fitness and functional status, these studies reporting moderate effect size for gait, postural balance, body flexibility, body strength, and weight loss [17]. In that context, we should investigate the most appropriate duration of yoga intervention and the most suitable postures and yoga styles for the older cohorts. All above mentioned studies require to employ sound methodology and thorough statistical analysis that will embrace (larger and randomized samples with more blinded studies). These studies need to be replicated in a variety of populations, both sick and well, as the effects may vary depending upon the health status of selected sample.

\section{Discussion}

Presented reviews point out a number of distinct areas where yoga may be beneficial. Research studies on yoga as a therapeutic intervention in relatively new field of study, therefore studies in related domains are rather small. We observe high heterogeneity in (group selection, type, frequency, intensity of intervention, selected measures, motivation of the participants, health status etc.). Therefore, better understanding of the significance of yoga under different circumstances is required. In contemporary society more and more people are turning to yoga for its mental benefits in relative to pharmaceutical, clinical intervention. Number of studies suggest that practicing yoga shows promise for promoting better population mental health. It appears that deep slow breathing in combination with movement and other aspects of yoga haw ability to bring person a greater sense of tranquility. We have base evidence that yoga can reduce symptoms of anxiety and depression $[18,19]$. However, additional investigation in this field are highly recommended, specifically because of the positive relevant psycho-physiological rationale. While it is not surprising that physical training can enhance physical fitness by the means of yoga or conventional training protocol. However, these effects are powerful in healthy individuals, still much weaker in individuals with chronic pain conditions. This facticity deserves further investigation. Motivation of participant stand out as an important, crucial factor that need to be emphasize. Compliance with yoga objectives, taking part in the class of yoga may be higher in social, community setting in comparison to performing yoga isolated, alone at home. However, intervention of short duration might be an option for some specific indications (i.e., pain, depression symptoms) while the cardiovascular and overall physical fitness effects might require long term practice.

Obviously, yoga intervention programs demand an active attendance, and therefore adherence might be a crucial point that can limits potentially beneficial effects of yoga. It is comprehensible that lifestyle play crucial role in health outcome. In that context plausible attribute of yoga intervention is that it may in fact be assistive for the execution and maintenance of such lifestyle changes due to the experience of well-being from the practice, which will in turn support desire to adopt and maintain healthy behaviors. Thus, further investigation should clarify which individual may benefit from the intervention and also which facet of the yoga intervention (i.e., physical activity, meditation, lifestyle alteration) or which particular yoga style is more effective. Yoga in its foundation has potential to be implemented as a safe and plausible treatment that is basically cost effective, enhancing self -confidence, self-esteem, self-efficacy, and is frequently associated with additional positive side effects.

The extent to which yoga intervention is curative treatment remains to be clarify. At the present time it is safe to suggest that yoga can be a beneficial supportive additive treatment. Yoga stands out as a low risk for side effects, when selecting appropriate postures and exercise protocol. However, the meditative, cognitive aspect of yoga could be questionable for the people with mental instability or personality disorder. We have not enough relevant evidence to claim, that yoga practice has negative impact on mental health. Even though, number of studies claiming positive benefits of yoga, multiple methodological limitations (heterogeneity of selected sample, small sample size, motivation of participants) bound the generalization of these ever-promising studies. We will need more, cross-sectional and longitudinal studies in order to determined, verify rather promising outcomes. In addition, we have to be aware, that yoga is not "exercise" but a "process. Future research is needed to examine the difference between yoga as an "exercise" and yoga as a "process", particularly, how these 


\section{Journal of Yoga and Physiotherapy}

two modalities may differ in the quest for better health. At the conclusion, yoga practice need to be further investigated as a tool to enhance physical fitness and hence quality of life.

\section{References}

1. Kirkwood G, Rampes H, Tuffrey V, Richardson J, Pilkington K (2005) Yoga for anxiety: a systematic review of the research evidence. British Journal of Sports Medicine 39(12): 884-891.

2. Boehm K, Ostermann T, Milazzo S, Büssing A (2012) Effects of yoga interventions on fatigue: a meta-analysis. Evid Based Complement Alternat Med 2012: 124703.

3. Khalsa SBS (2004) Yoga as a therapeutic intervention: a bibliometric analysis of published research studies. Indian J Physiol Pharmacol 48(3): 269-285.

4. Saeed SA, Antonacci DJ, Bloch RM (2010) Exercise, yoga, and meditation for depressive and anxiety disorders. American Family Physician 81(8): 981-986.

5. Pilkington K, Kirkwood G, Rampes H, Richardson J (2005) Yoga for depression: the research evidence. Journal of Affective Disorders 89(13): 13-24.

6. Innes KE, Bourguignon C, Taylor AG (2005) Risk indices associated with the insulin resistance syndrome, cardiovascular disease, and possible protection with yoga: a systematic review. Journal of the American Board of Family Practice 18(6): 491-519.

7. Aljasir B, Bryson M, Al-Shehri A (2010) Yoga practice for the management of type II diabetes mellitus in adults: a systematic review. Evidence-Based Complementary and Alternative Medicine 7(4): 399-408.

8. Singh V, Wisniewski A, Britton J, Tattersfield A (1990) Effect of yoga breathing exercises (pranayama) on airway reactivity in subjects with asthma. Lancet 335(8702): 1381-1383.
9. Büssing A, Ostermann T, Lüdtke R, Michalsen A (2012) Effects of yoga interventions on pain and pain-associated disability: a metaanalysis. Journal of Pain 13(1): 1-9.

10. Posadzki P, Ernst E, Terry R, Lee MS (2011) Is yoga effective for pain? A systematic review of randomized clinical trials. Complementary Therapies in Medicine 19(5): 281-287.

11. Ross A (2010) The Health Benefits of Yoga and Exercise: A review of Comparison Studies. Journal of alternative and complementary medicine 16(1): 3-12.

12. Hagins M, Moore W, Rundle A (2007) Does practicing hatha yoga satisfy recommendations for intensity of physical activity which improves and maintains health and cardiovascular fitness? BMC Complement Altern Med 7: 1-9.

13. Tran MD, Holly RG, Lashbrook J, Amsterdam EA (2001) Effects of hatha yoga practice on health-related aspects of physical fitness. PrevCardiol 4(4): 165-170.

14. Few JD (1974) Effect of exercise on the secretion and metabolism of cortisol in man. J Endocrin 62(2): 341-353.

15. Bhutkar MV, Bhutkar PM, Taware GB, Surdi AD (2011) How Effective is Sun Salutation in Improving Muscle Strength, General Body Endurance and Body Composition? Asian J Sports Med 2(4): 259-266.

16. Clay CC, Lloyd LK, Walker JL, Sharp KR, Pankey RB (2005) The metabolic cost of hatha yoga. J Strength Cond Res 19(3): 604-610.

17. Roland KP, Jakobi JM, Jones GR (2011) Does yoga engender fitness in older adults? A critical review. Journal of Aging and Physical Activity 19(1): 62-79.

18. Shroff FM, Asgarpour M (2017) Yoga and Mental Health: A Review. Journal of Physiotherapy and Rehabilitation 2: 1 .

19. Rakhshani A, Maharana S, Raghuram N, Nagendra HR, Venkatram $P$ (2010) Effects of integrated yoga on quality of life and interpersonal relationship of pregnant women. Qual Life Res 19: 1447-1455.

\section{Your next submission with Juniper Publishers} will reach you the below assets

- Quality Editorial service

- Swift Peer Review

- Reprints availability

- E-prints Service

- Manuscript Podcast for convenient understanding

- Global attainment for your research

- Manuscript accessibility in different formats

(Pdf, E-pub, Full Text, Audio)

- Unceasing customer service

Track the below URL for one-step submission

https://juniperpublishers.com/online-submission.php 